\title{
Airfoil Optimization Design by Panel Methods for Small Size Aerial Vehicle at Low Reynolds Number
}

\author{
LIU Wu-fa \\ School of Mechanical Engineering \\ Zhengzhou University, ZZU \\ Zhengzhou, China, +8637167781235 \\ wufaliu126@126.com
}

GONG Zhen-bang

School of Mechatronic Engineering and Automation Shanghai University, SHU

Shanghai, China

\author{
LI Su-yan \\ School of Mechanical Engineering \\ Zhengzhou University, ZZU \\ Zhengzhou, China
}

\author{
JIANG Zhen \\ School of Mechatronic Engineering and Automation \\ Shanghai University, SHU \\ Shanghai, China
}

\begin{abstract}
Airfoil optimization design has been carried out by panel methods for small size aerial vehicle at low Reynolds number. The optimization airfoil formulas were expressed by the NACA4 digital airfoil analytical expressions with a few optimization variables that made the optimizing computation quick. A system of $n+1$ dimensional linear equations have been derived from vortex panel and source panel methods to compute the vortex and source strengths on the airfoil surface and aerodynamic coefficients in airfoil optimization process. The airfoil was optimized under the condition of taking the maximum lifting coefficient of taking off or landing without stall for optimization goal by Matlab optimization toolbox. The aerodynamic analysis results of optimized airfoil by XFOIL indicate that the lifting coefficients all have some extent increase relative to drag coefficients in the range of 0-12 degrees of angle of attack respectively.
\end{abstract}

Keywords- low Reynolds number; airfoil optimization; panel method; lifting coefficient

\section{INTRODUCTION}

Aerial vehicle Airfoil has important influence on the wing's aerodynamic characteristics. Therefore, airfoil design is very important. Airfoil aerodynamic coefficients are all need to calculate in its forward and reverse designs. The main computing theories are Thin Airfoil Theory [1], KutaJoukowski theorem [2], Vortex Panel Method [3], Conformal Mapping Method [4], etc.

Hicks [5] thought, from the practical view of consideration, aerodynamic optimization design was very attractive, because this method could solve a lot of design problems.

For a plane with wing span of 2.0 meters, preliminary design showed that wing root chord is about $0.405 \mathrm{~m}$, wing tip chord is about $0.22 \mathrm{~m}$, and its takeoff speed is about $35 \mathrm{~km} / \mathrm{h}$, the highest cruising speed is about $80 \mathrm{~m} / \mathrm{h}$. Therefore, its average chord Reynolds number from 208000 to 475400 should be in the range of low Reynolds number. Based on the basic airfoil, this paper is to design the airfoil with optimization theory, and analyzes its aerodynamic performance at low Reynolds number.

\section{AIRFOIL FORMULA}

Good results had been obtained by optimizing airfoils in [69] with airfoil representations by type functions or spline curves. However, they usually need more than 20 design variables to represent airfoil accurately, and need large amount of calculation, and had low optimization efficiency [10]. When using analytic function to express airfoil, such as NACA series, 5 design variables are enough to express airfoil geometry shape, and optimization calculation speed is high.

The normal analytic equation for NACA 4 digits airfoil is as follows

$$
\begin{aligned}
y_{t}= & 5 t(0.2969 \sqrt{x}-0.1260 x \\
& \left.-0.3516 x^{2}+0.2843 x^{3}-0.1015 x^{4}\right)
\end{aligned}
$$

Airfoil bending analytic equation is as follows

$$
\begin{gathered}
y_{c}=\frac{m}{p^{2}}\left(2 p x-x^{2}\right),(0 \leq x \leq p) . \\
y_{c}=\frac{m}{(1-p)^{2}}\left[(1-2 p)+2 p x-x^{2}\right],(p \leq x \leq c) .
\end{gathered}
$$

where $y_{t}$ is coordinate in airfoil thickness direction; $x$ is coordinate in airfoil length direction, from 0 to $c ; y_{c}$ is airfoil bending coordinate; $t$ is airfoil maximum thickness; $m$ is airfoil maximum bending; $p$ is is maximum bending position. $t, m$, and $p$ are all expressed by a percentage of the wing root chord.

$\left(x_{u}, y_{u}\right)$ are the airfoil upper surface coordinates, $\left(x_{l}, y_{l}\right)$ are lower surface coordinates, the airfoil can be expressed as

$$
\left\{\begin{array}{l}
x_{u}=x-y_{t} \sin \theta \\
y_{u}=y_{c}+y_{t} \cos \theta \\
x_{l}=x+y_{t} \sin \theta \\
y_{l}=y_{c}-y_{t} \cos \theta \\
\theta=\arctan \left(d y_{c} / d x\right)
\end{array} .\right.
$$

The National Hightech Plan Project (2001AA422160) 
Equation (1), (2) and (3) substituted to (4), NACA4 series airfoil analytic expressions can get in (5) and (6).

$$
\begin{aligned}
& \left\{\begin{array}{l}
x_{u}=x-5 t\left(0.2969 \sqrt{x}-0.1260 x-0.3516 x^{2}+0.2843 x^{3}-0.1015 x^{4}\right) \sin \theta \\
y_{u}=\frac{m}{p^{2}}\left(2 p x-x^{2}\right)+5 t\left(0.2969 \sqrt{x}-0.1260 x-0.3516 x^{2}+0.2843 x^{3}-0.1015 x^{4}\right) \cos \theta \\
x_{l}=x+5 t\left(0.2969 \sqrt{x}-0.1260 x-0.3516 x^{2}+0.2843 x^{3}-0.1015 x^{4}\right) \sin \theta \\
y_{l}=\frac{m}{p^{2}}\left(2 p x-x^{2}\right)-5 t\left(0.2969 \sqrt{x}-0.1260 x-0.3516 x^{2}+0.2843 x^{3}-0.1015 x^{4}\right) \cos \theta \\
\theta=\arctan \left(d y_{c} / d x\right) \\
0 \leq x \leq p
\end{array}\right. \\
& \left\{\begin{array}{l}
x_{u}=x-5 t\left(0.2969 \sqrt{x}-0.1260 x-0.3516 x^{2}+0.2843 x^{3}-0.1015 x^{4}\right) \sin \theta \\
y_{u}=\frac{m}{(1-p)^{2}}\left[(1-2 p)+2 p x-x^{2}\right]+5 t\left(0.2969 \sqrt{x}-0.1260 x-0.3516 x^{2}+0.2843 x^{3}-0.1015 x^{4}\right) \cos \theta \\
x_{l}=x+5 t\left(0.2969 \sqrt{x}-0.1260 x-0.3516 x^{2}+0.2843 x^{3}-0.1015 x^{4}\right) \sin \theta \\
y_{l}=\frac{m}{(1-p)^{2}}\left[(1-2 p)+2 p x-x^{2}\right]-5 t\left(0.2969 \sqrt{x}-0.1260 x-0.3516 x^{2}+0.2843 x^{3}-0.1015 x^{4}\right) \cos \theta \\
\theta=\arctan \left(d y_{c} / d x\right) \\
p \leq x \leq c
\end{array}\right.
\end{aligned}
$$

\section{OPTIMIZATION OBJECTIVE FUNCTION}

According to (5) and (6), NACA4 airfoils are determined by variables $m, p$ and $t$, thus, they can be as optimization variables. In order to ensure wing strength, the airfoil maximum thickness should not be changed or changed little. Therefore, NACA2415 is chosen as the basic airfoil, $m$ and $p$ are selected as optimization variables.

The lifting coefficient of the NACA2415 airfoil is about 0.18 at zero angle of attack, and the maximum lifting coefficient is about 1.0 at 10 degree angle of attack. The purpose of the optimization is to increase the maximum lifting coefficient to 1.1 to ensure landing and take off speed without stall. The optimizing objective function and constraints are as follows.

$$
\begin{aligned}
& \min F=\left(C_{l}-1.1\right)^{2} \\
& \text { s.t. }\left\{\begin{array}{c}
0.0 \leq m \leq 0.05 \\
0.1 \leq p \leq 0.5
\end{array}\right.
\end{aligned}
$$

Equation (7) can realize by the optimization function $f_{\text {mincon }}$ in MATLAB Toolbox.

\section{Aerodynamics Computering By PANel Methods}

Panel methods can deal with the computation of complex aerodynamic layout, such as Boeing 747, the computing speed and reliability were admired by aircraft designers. So, this article uses vortex panel method [11] to calculate pressure coefficients to optimize airfoil.

The vortex sheet is approximated by a series of straight panels shown in Fig. 1. Let the vortex strength $\gamma(s)$ per unit length be constant over a given panel, but allow it to vary from one panel to the next. That is, for the $n$ panels shown in Fig. 1, the vortex panel strength per unit length are $\gamma_{1}, \gamma_{2}, \cdots, \gamma_{j}, \cdots, \gamma_{n}$. These panel strengths are unknowns, the main purpose of the panel technique is to solve for $\gamma_{j}, j=1$ to $n$, such that the body surface becomes a streamline of the flow and such that the Kutta condition is satisfied. The midpoint of each panel is a control point at which the boundary condition is applied, that is, at each control point, the normal component of the flow velocity is zero.

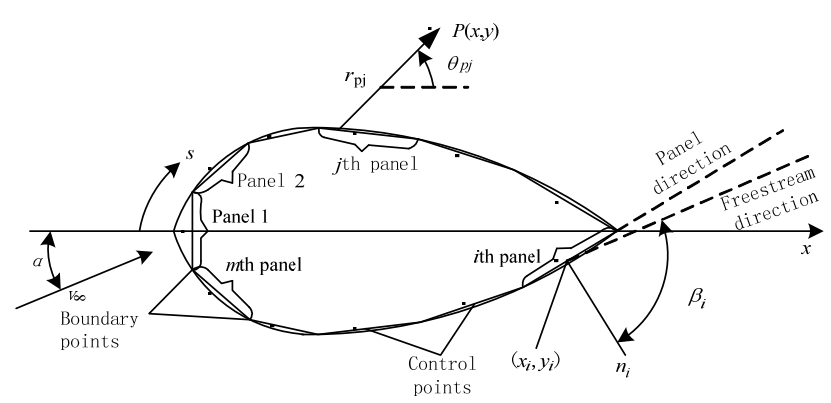

Figure 1. Vortex distribution over the surface of a body of arbitrary shape

Let $P$ be a point located at $(x, y)$ in the flow, and let $r_{p j}$ be the distance from any point on the $j$ th panel to $P$, as shown in Fig. 1 . The radius $r_{p j}$ makes the angle $\theta_{p j}$ with respect to the $x$ axis.

The normal component of the flow velocity at the $i$ th control point is the sum of that due to the freestream and that due to the vortex panels. The boundary condition states that this sum must be zero:

$$
V_{\infty, n}+V_{n}=0 .
$$

By the velocity potential and vortex panels, we obtain

$$
V_{\infty} \cos \beta_{i}-\sum_{j=1}^{n} \frac{\gamma_{j}}{2 \pi} \int_{j} \frac{\partial \theta_{i j}}{\partial n_{i}} d s_{j}=0 .
$$

Equation (8) is the key of the vortex panel method. The values of the integrals in (8) depend simply on the panel geometry, they are not properties of the flow. Let $J_{i, j}$ be the value of this integral when the control point is on the $i$ th panel. (8) could be written as

$$
V_{\infty} \cos \beta_{i}-\sum_{j=1}^{n} \frac{\gamma_{j}}{2 \pi} J_{i, j}=0 .
$$


Equation (9) is a linear algebraic equation with $n$ unknowns, $\gamma_{1}, \gamma_{2}, \cdots, \gamma_{n}$. It represents the flow boundary condition evaluated at the control point of the $i$ th panel. If (9) is applied to the control points of all the panels, we obtain a system of $n$ linear equations with $n$ unknowns.

Because Kutta condition $\left(\gamma_{i}=-\gamma_{i-1}\right)$ eliminates a equation at the trailing edge, when (9) applied to the control points of all the panels, we can constitute one underdetermined system of $n$ unknowns with $n-1$ equations. There are several ways to solve this problem, what is more common usually is to use a combination of the vortex panels and source panels [12] in a panel solution, the source panels are used to simulate the airfoil thickness, and the vortex panels are used to cause circulation. When this combination is adopted, we get a system of $n$ linear algebraic equations with $n+1$ unknowns, $\gamma_{1}, \gamma_{2}, \ldots, \gamma_{n}$ and $\lambda$ (source strength per unit length). We obtain

$$
\sum_{i=1}^{n} V_{\infty} \cos \beta-\sum_{n=1}^{n} \sum_{j=1}^{n} \frac{\gamma_{j}}{2 \pi} J_{i, j}+\sum_{i=1}^{n} \sum_{j=1}^{n} \frac{\lambda}{2 \pi} I_{i, j}=0 .
$$

The total tangential velocity is

$$
V_{t}=V_{\infty} \sin \beta_{i}-\sum_{j=1}^{n} \frac{\gamma_{j}}{2 \pi} A_{i, j}+\sum_{j=1}^{n} \frac{\lambda}{2 \pi} B_{i, j}
$$

The panels on the airfoil are configured as described in Fig. 2 , the tangential velocity of the control point on the first panel which can be deduced from (11) is

$$
V_{1}=V_{\infty} \sin \beta_{1}-\sum_{j=1}^{n} \frac{\gamma_{j}}{2 \pi} A_{1, j}+\sum_{j=1}^{n} \frac{\lambda}{2 \pi} B_{1, j}
$$

The tangential velocity of the control point on the nth panel which can be deduced from (11) is

$$
V_{n}=V_{\infty} \sin \beta_{n}-\sum_{j=1}^{n} \frac{\gamma_{j}}{2 \pi} A_{n, j}+\sum_{j=1}^{n} \frac{\lambda}{2 \pi} B_{n, j}
$$

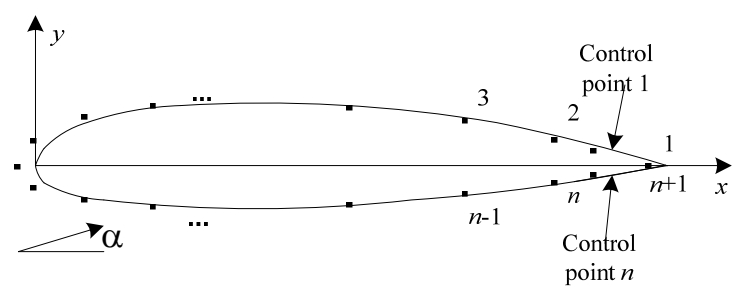

Figure 2. Panels configuration of the airfoil

Now, Kutta condition can be converted to $V_{1}+V_{n}=0$, and substituting (12) and (13) into this condition, Kutta condition can also be expressed as

$$
\begin{aligned}
& -\frac{1}{2 \pi}\left[\gamma_{1}\left(A_{1,1}+A_{n, 1}\right)+\gamma_{2}\left(A_{1,2}+A_{n, 2}\right)+\cdots+\gamma_{n}\left(A_{1, n}+A_{n, n}\right)\right] \\
& +\frac{1}{2 \pi} \lambda\left[\sum_{j=1}^{n} B_{1, j}+\sum_{j=1}^{n} B_{n, j}\right]=-V_{\infty}\left(\sin \beta_{1}+\sin \beta_{n}\right)
\end{aligned}
$$

By (10) and (14), we obtain a determined system of $n+1$ linear algebraic equations with $n+1$ unknowns, $\gamma_{1}, \gamma_{2}, \ldots, \gamma_{n}$ and $\lambda$.

$$
\frac{1}{2 \pi}\left[\begin{array}{ccccc}
J_{1,1} & J_{1,2} & \cdots & J_{1, n} & -\sum_{j=1}^{n} I_{1, j} \\
J_{2,1} & j_{2,2} & \cdots & J_{2, n} & -\sum_{j=1}^{n} I_{2, j} \\
\vdots & \vdots & \cdots & \vdots & \vdots \\
J_{n, 1} & J_{n, 2} & \cdots & J_{n, n} & -\sum_{j=1}^{n} I_{n, j} \\
A_{1,1}+A_{n, 1} & A_{1,2}+A_{n, 2} & \cdots & A_{1, n}+A_{n, n} & -\sum_{j=1}^{n} B_{1, j}-\sum_{j=1}^{n} B_{n, j}
\end{array}\right]\left[\begin{array}{c}
\gamma_{1} \\
\gamma_{2} \\
\vdots \\
\gamma_{n n} \\
\lambda
\end{array}\right]=V_{\infty}\left[\begin{array}{c}
\cos \beta_{1} \\
\cos \beta_{2} \\
\vdots \\
\cos \beta_{n} \\
\sin \beta_{1}+\sin \beta_{n}
\end{array}\right]
$$

Where $J_{i, j}=\int_{j} \frac{\partial \theta_{i j}}{\partial n_{i}} d s_{j}, A_{i, j}=\int_{j} \frac{\partial \theta_{i j}}{\partial s} d s_{j}, I_{i, j}=\int_{j} \frac{\partial}{\partial n_{i}}\left(\ln r_{i j}\right) d s_{j}$, $B_{i, j}=\int_{j} \frac{\partial}{\partial s}\left(\ln r_{i j}\right) d s_{j}$.

Now, for a solution of the given airfoil geometry, that is, angle of attack $\alpha$ and freestream velocity $V_{\infty}$ and panels configuration, then $\beta_{i}$ can be computed out by Fig. 1, consequently we solve (15) to get $\gamma_{1}, \gamma_{2}, \ldots, \gamma_{n}$ and $\lambda$, and finally the pressure coefficient at the $i$ th control point is obtained from (16)

$$
C_{P, i}=1-\left(\frac{\gamma_{i}}{V_{\infty}}\right)^{2}
$$

Through the integral of the pressure coefficients of upper and lower airfoil surfaces, we can get airfoil normal force coefficient $c_{n}$, tangential force coefficient $c_{\alpha}$ and moment coefficient $c_{m}$, and then the lifting and drag coefficients can be calculate out by (17).

$$
\left\{\begin{array}{l}
c_{l}=c_{n} \cos \alpha-c_{\alpha} \sin \alpha \\
c_{d}=c_{n} \sin \alpha+c_{\alpha} \cos \alpha
\end{array}\right.
$$

\section{OPTIMIZATION RESUlts, ANALYSIS AND VERIFICATION}

In this paper, MATLAB 6.5 is used to program optimization algorithm and aerodynamic coefficient calculation of panel methods.

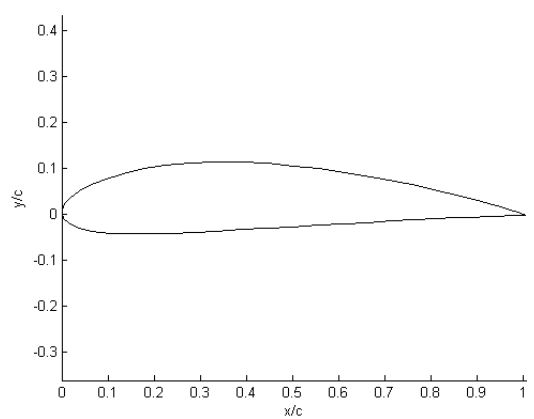

Figure 3. Optimized airfoil

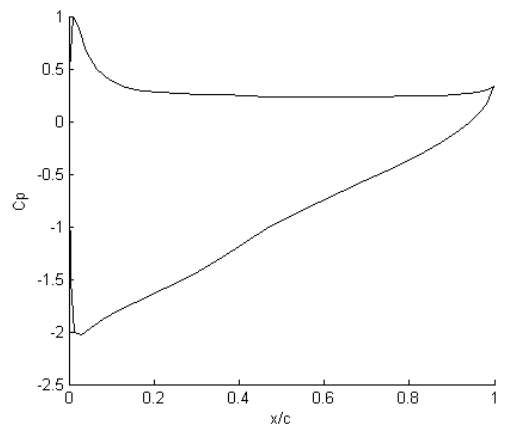

Figure 4. Surface pressure distributions of optimized airfoil 
Fig. 3 is the optimized airfoil, Fig. 4 is the pressure distribution of the optimized airfoil. The optimized design variables, $m, p$ are $0.0401,0.3991$ respectively.

The optimized airfoil can satisfy the take-off and landing, and the pressure distribution on the upper and lower airfoil surfaces is reasonable. Moreover, from the point of view of manufacturing technology, there does not exist concave on the airfoil.
XFOIL [13] is one application software for airfoil analysis and reverse design written by Harold Youngren, Aerocraft, Inc., MIT Aero \& Astro based on the panel methods. In the condition of $60 \mathrm{~km} / \mathrm{h}$ velocity, XFOIL analysis results for NACA2415 and the optimized airfoil are shown in table I, we can see that lifting coefficients are added from 0 to 12 degree of angle of attack with respect to the drag coefficients.

TABLE I. RESULTS COMPARISON FOR NACA2415 AND THE OPTIMIZED AIRFOIL BY XFOIL

\begin{tabular}{|c|c|c|c|c|c|c|c|c|}
\hline \multirow[b]{2}{*}{$\alpha\left({ }^{\circ}\right)$} & \multicolumn{3}{|c|}{ NACA2415 } & \multicolumn{3}{|c|}{ Optimized airfoil } & \multirow[b]{2}{*}{$\Delta C_{l} \%$} & \multirow[b]{2}{*}{$\Delta C_{d} \%$} \\
\hline & $C_{l}$ & $C_{d}$ & $C_{m}$ & $C_{l}$ & $C_{d}$ & $C_{m}$ & & \\
\hline 0 & 0.233 & 0.0082 & -0.049 & 0.429 & 0.0089 & -0.088 & 84.1 & 8.53 \\
\hline 2 & 0.456 & 0.0089 & -0.046 & 0.811 & 0.0101 & -0.119 & 77.9 & 13.5 \\
\hline 4 & 0.763 & 0.0102 & -0.064 & 0.942 & 0.0111 & -0.101 & 23.5 & 8.82 \\
\hline 6 & 0.921 & 0.0116 & -0.051 & 1.083 & 0.0124 & -0.084 & 17.6 & 6.90 \\
\hline 8 & 1.072 & 0.0143 & -0.038 & 1.191 & 0.0139 & -0.062 & 11.1 & -2.80 \\
\hline 10 & 1.189 & 0.0189 & -0.022 & 1.277 & 0.0177 & -0.040 & 7.40 & -6.35 \\
\hline 12 & 1.281 & 0.0259 & -0.008 & 1.328 & 0.0259 & -0.021 & 3.67 & 0.00 \\
\hline
\end{tabular}

\section{CONCLUSIONS}

First of all, this paper analyzed the representations of airfoil and its optimization methods, and put forward the airfoil optimization variables $m, p$, and established the objective function and constraints.

Then, according to the theory and method of the Vortex panel and Source panel, calculation method of the airfoil aerodynamic coefficients was established.

Furthermore, according to optimization function and aerodynamic coefficient calculation method, the airfoil has been optimized, the optimized airfoil not only meet the take-off and landing requirements, but also is suitable for manufacturing.

Finally, the aerodynamic coefficient comparison between NACA2415 airfoil and the optimized airfoil showed that the optimized airfoil aerodynamic performance is improved.

\section{REFERENCES}

[1] John R. and Alberta Y. Alksne. "Thin airfoil theory tased on approximate solution of the transonic flow equation," NACA Technical Note 3970.

[2] H.Schlichting, E.Truckenbordt. Aerodynamics of airplane. Beijing: National Industry Press, 1978, pp. 380 384 (in Chinese).
[3] http: //www.ae.su.oz.au/aero/panel2d/ panel2d.html.

[4] H.Schlichting, E.Truckenbordt. Aerodynamics of airplane. Beijing: National Industry Press, 1978, pp. 384 402 (in Chinese).

[5] R.M. Hicks, E.M. Murman, and G.N. Van-derplaats. "An assessment of airfoil design by numerical optimization,” NASA TM X-3092, 1974.

[6] G. Kuruvila, Shlomo Ta'asan, and M.D.Salas. "Airfoil design and optimization by the one-shot method," 33rd Aerospace Sciences Meeting and Exhibit, Reno, NV. January 9-12, 1995, AIAA 95-0478.

[7] William E. Milholen II. "An efficient inverse aerodynamic design method for subsonic flows," 38 Aerospace Sciences Meeting and Exhibit, Reno, NV. January10-13 2000, AIAA2000-0780.

[8] M. Drela. "XFOIL: An analysis and design system for low Reynolds number airfoils," Conference on Low Reynolds Number Airfoil Aerodynamics, University of Notre Dame, June 1989.

[9] Kristian S.Dahl,Peter Fuglsang. "Design of the wind turbine airfoil family RISØ-A-XX.RisØ," National Laboratory, Roskilde, Denmark, December 1998.

[10] Yushin Kiin and Kwanjung Yee. "Aerodynamic shape design of rotor airfoils undergoing unsteady motion," Journal of Aircraft, March-April 2004,Vol.41,No.2, pp.247-257.

[11] Jr. Anderson, D. John. Fundamentals of aerodynamics: Lifting flows over arbitrary bodies-The vortex panel numerical method. 3nd., McGraw-Hill Inc., New York, 2001, pp.319-324.

[12] Jr. Anderson, D. John. Fundamentals of aerodynamics: Nonlifting flows over arbitrary bodies-The numerical source panel method. 3nd., McGraw-Hill Inc., New York, 2001, pp.247-256.

[13] Mark Drela. XFOIL 6.94 User Guide. MIT Aero \& Astro, Harold Youngren Aerocraft, Inc., 10 Dec. 2001. 